\author{
LINEAR AND NON-LINEAR THEORY OF \\ GENERALIZED FUNCTIONS AND ITS APPLICATIONS \\ BANACH CENTER PUBLICATIONS, VOLUME 88 \\ INSTITUTE OF MATHEMATICS \\ POLISH ACADEMY OF SCIENCES \\ WARSZAWA 2010
}

\title{
THE ALGEBRA OF POLYNOMIALS ON THE SPACE OF ULTRADIFFERENTIABLE FUNCTIONS
}

\author{
KATARZYNA GRASELA \\ Institute of Mathematics, Cracow University of Technology \\ Warszawska 24, 31-155 Kraków, Poland \\ E-mail: katarzyna.grasela@gmail.com
}

\begin{abstract}
We consider the space $\mathcal{D}^{\mathcal{M}}$ of ultradifferentiable functions with compact supports and the space of polynomials on $\mathcal{D}^{\mathcal{M}}$. A description of the space $\mathcal{P}\left(\mathcal{D}^{\mathcal{M}}\right)$ of polynomial ultradistributions as a locally convex direct sum is given.
\end{abstract}

1. Introduction. Roumieu and Beurling ultradistributions are meant as elements of the dual space to a non-quasi analytic class of infinitely differentiable functions equipped with a natural locally convex topology (see e.g. 9]). In this paper, we will consider the space $\mathcal{P}\left(\mathcal{D}^{\mathcal{M}}\right)$ of polynomial ultradistributions, where $\mathcal{D}^{\mathcal{M}}$ denotes the space of ultradifferentiable functions (for the definition see Section 2). The space $\mathcal{P}\left(\mathcal{D}^{\mathcal{M}}\right)$ contains the space of ultradistributions as a proper subspace and it is the smallest space, which is stable under tensor multiplication. We shall describe the space $\mathcal{P}\left(\mathcal{D}^{\mathcal{M}}\right)$ in terms of the direct sums of symmetric tensor powers of the space $\mathcal{D}_{\mathcal{M}}^{\prime}$, dual to $\mathcal{D}^{\mathcal{M}}$; we prove that such a direct sum is a convolution algebra. In physics such algebras are known as Borcher's algebras (cp. [1]). It is widely known that the space $\mathcal{D}^{\mathcal{M}}\left(R^{n}\right)$ of ultradifferentiable functions equipped with a natural locally convex topology is topologically isomorphic to the space $E\left(C^{n}\right)$ of entire functions of exponential type [4, via the Fourier-Laplace transformation; we shall prove, however, that this isomorphism can be extended to the corresponding spaces of polynomials.

2. Polynomials on locally convex spaces. In this paper the symbol $N_{1}$ denotes the set $N \backslash\{0\}$ of strictly positive integer numbers.

Let $\mathcal{L}^{n}(X, C)$ denote the space of $n$-linear, continuous forms defined on a locally

2000 Mathematics Subject Classification: Primary 46F25; Secondary 46F10, 44A10.

Key words and phrases: ultradistributions of Roumieu type, polynomial ultradistributions, entire functions of exponential type.

The paper is in final form and no version of it will be published elsewhere. 
convex space $X$

$$
F_{n}: \prod_{\imath=1}^{n} X:=\underbrace{X \times \ldots \times X}_{n} \ni\left(x_{1}, \ldots, x_{n}\right) \mapsto F_{n}\left(x_{1}, \ldots, x_{n}\right) \in C .
$$

With any $n$-linear, continuous form $F_{n} \in \mathcal{L}^{n}(X, C)$ we can associate the composition

$$
P_{n}=F_{n} \circ \Delta_{n}, \quad \Delta_{n}: X \ni x \mapsto{ }^{n} x:=(x, \ldots, x) \in \prod_{\imath=1}^{n} X,
$$

which, according to [2], we shall call a homogenous polynomial of degree $n$ on the space $X$. The linear space of all homogenous polynomials of degree $n$ will be denoted by $\mathcal{P}_{n}(X)$.

When we have a polynomial $P_{n} \in \mathcal{P}_{n}(X)$ we can get back the linear symmetric form $F_{n}$, associated to $P_{n}$, by the following polarization formula (comp. i.e [2])

$$
F_{n}\left(x_{1}, \ldots, x_{n}\right)=\frac{1}{2^{n} n !} \sum_{e_{\imath}= \pm 1} e_{1} \ldots e_{n} P_{n}\left(\sum_{\imath=1}^{n} e_{\imath} x_{\imath}\right) .
$$

On the space $\mathcal{L}^{n}(X, C)$ we will consider the locally convex topology of uniform convergence on bounded, absolutely convex subsets of $\prod_{\imath=1}^{n} X$, this topology will be denoted by $\tau_{\beta}$. By $\tau_{\beta}$ we will also denote the topology on the space $\mathcal{P}_{n}(X)$ of uniform convergence on bounded, absolutely convex subsets of $X$.

By the algebra of polynomials on the space $X$, we mean the locally convex direct sum

$$
\mathcal{P}(X):=\sum_{n \in N_{1}} \mathcal{P}_{n}(X)=\left\{P(x)=\sum_{n=1}^{m} P_{n}(x): P_{n} \in \mathcal{P}_{n}(X) ; m \in N_{1}\right\} .
$$

It is obvious that $\mathcal{P}(X)$ is an algebra with respect to multiplication

$$
\begin{aligned}
& \mathcal{P}(X) \times \mathcal{P}(X) \ni(P, Q) \mapsto P Q \in \mathcal{P}(X), \\
& P(x) Q(x)=\sum_{n \in N_{1}} \sum_{m=1}^{n} P_{m}(x) Q_{n-m+1}(x), \quad x \in X .
\end{aligned}
$$

Now, we would like to introduce some notations, connected with tensor products. Let $\otimes^{n} X:=X \otimes \ldots \otimes X$ denote the algebraic tensor product of $n$ copies of the space $X$, and let $\widehat{\otimes}_{p}^{n} X$ denote its completion in the projective topology. In the space $\otimes^{n} X$ we consider the operation of symmetrization

$$
\varsigma_{n}: \otimes^{n} X \ni x_{1} \otimes \ldots \otimes x_{n} \mapsto x_{1} \odot \ldots \odot x_{n}:=\frac{1}{n !} \sum_{\varsigma \in G_{n}} x_{\varsigma(1)} \otimes \ldots \otimes x_{\varsigma(n)},
$$

where $G_{n}$ is the group of permutations.

The operator $\varsigma_{n}$ is a projection in the space $\otimes^{n} X$, continuous with respect to the given topology $\tau\left[2\right.$, hence it can be extended onto the completion of $\otimes^{n} X$. This extension will be also denoted by $\varsigma_{n}$. In our paper by

$$
\left(x_{1} \otimes \ldots \otimes x_{m}\right) \odot\left(x_{m+1} \otimes \ldots \otimes x_{n}\right), \quad 1 \leq m \leq n,
$$

we shall understand $x_{1} \odot x_{2} \odot \ldots \odot x_{n}$ and the operator $\odot$ can be extended by linearity and continuity to an operator $\left(\widehat{\otimes}_{p}^{m} X\right) \times\left(\widehat{\otimes}_{p}^{n-m} X\right) \rightarrow \widehat{\odot}_{p}^{n} X$.

We shall use the following notation: $\odot^{n} X:=\varsigma_{n}\left(\otimes^{n} X\right)$, and $\widehat{\odot}_{p}^{n} X:=\varsigma_{n}\left(\widehat{\otimes}_{p}^{n} X\right)$. 
Let $\chi_{n}$ denote the canonical inclusion of the cartesian product into the tensor product

$$
\chi_{n}: \prod_{i=1}^{n} X \ni\left(x_{1}, \ldots, x_{n}\right) \mapsto x_{1} \otimes \ldots \otimes x_{n} \in \otimes^{n} X .
$$

3. The space $D^{\mathcal{M}}$ and its properties. Let us consider $N_{1}^{n}$ with lexicographical order and by $\bar{k}, \widehat{k}$ we will denote the predecessor and the successor of $k$ for $k \in N_{1}^{n}$.

Let $\mathcal{M} \equiv\left\{\mu_{k}\right\}_{k \in N_{1}^{n}}$ denote a sequence of positive numbers with the following properties:

(1M) $\mu_{k}^{2} \leq \mu_{\bar{k}} \mu_{\widehat{k}},($ logarithmic convexity);

(2M) $\sum_{k \in N_{1}^{n}} \frac{\mu_{k}}{\mu_{\widehat{k}}}<\infty$ (non-quasi analyticity);

(3M) there are $c>0$ and $d_{j}>0(j=1, \ldots, n)$ such that $\mu_{\widehat{k}} \leq c d^{k} \mu_{k}$, where $d=$ $\left(d_{1}, \ldots, d_{n}\right)$ (stability under differential operators)

If for $a, b \in R^{n}$ such that $a_{j}<b_{j}(j=1, \ldots, n),[a, b]$ denotes the $n$-dimensional interval $\prod_{j=1}^{n}\left[a_{j}, b_{j}\right]$ and $\nu \in \operatorname{int} R_{+}^{n}$ is any vector with positive coordinates, then we will consider the following space

$$
D_{[a, b], \nu}^{M}\left(R^{n}\right):=\left\{\varphi \in C^{\infty}\left(R^{n}\right): \operatorname{supp} \varphi \subset[a, b],\|\varphi\|_{[a, b], \nu}<\infty\right\}
$$

where

$$
\|\varphi\|_{[a, b], \nu}:=\sup _{t \in[a, b]} \sup _{k \in N_{1}^{n}}\left|\frac{D^{k} \varphi(t)}{\nu^{k} \mu_{k}}\right|
$$

with $D^{k}=D_{1}^{k_{1}} \ldots D_{n}^{k_{n}}, \quad D_{j}^{k_{j}}=\left(-i \frac{\partial}{\partial t_{j}}\right)^{k_{j}}$ and $\nu^{k}=\nu_{1}^{k_{1}} \ldots \nu_{n}^{k_{n}}$.

Let us define an order relation between vectors of $R^{n}$, namely $a \succ b$ if and only if $a_{j}<b_{j}, j=1, \ldots, n$.

By $D^{\mathcal{M}}\left(R^{n}\right)$ we mean the inductive limit of the spaces $D_{[a, b], \nu}^{M}\left(R^{n}\right)$, i.e.

$$
D^{\mathcal{M}}\left(R^{n}\right)=\operatorname{limind}_{\nu \succ 0, a \succ b} D_{[a, b], \nu}^{M}\left(R^{n}\right)
$$

with the inductive limit topology.

The Denjoy-Carleman Theorem implies that $D^{\mathcal{M}}\left(R^{n}\right)$ is nontrivial. If by $D_{\mathcal{M}}^{\prime}\left(R^{n}\right)$ we denote the dual space for $D^{\mathcal{M}}\left(R^{n}\right)$ then the following properties of $D^{\mathcal{M}}\left(R^{n}\right)$ and $D_{\mathcal{M}}^{\prime}\left(R^{n}\right)$ hold (see [4, Theorem 2.6])

THEOREM 3.1.

(i) Every $D_{[a, b], \nu}^{\mathcal{M}}\left(R^{n}\right)$ is a Banach space.

(ii) The inclusions

$$
D_{[a, b], \nu}^{M}\left(R^{n}\right) \mapsto D_{[c, d], \mu}^{M}\left(R^{n}\right), \quad[a, b] \subset[c, d], \quad \nu \prec \mu
$$

are compact.

(iii) $D^{\mathcal{M}}\left(R^{n}\right)$ is a nuclear, reflexive, (DF)-space.

(iv) $D_{\mathcal{M}}^{\prime}\left(R^{n}\right)$ is a nuclear, reflexive, $(F)$-space and an $\left(M^{*}\right)$ space in the sense of Silva. 


\section{The description of the space of polynomials on the space of ultradifferen-}

tiable functions. In order to simplify the notation by $\mathcal{D}^{\mathcal{M}}$ we will denote the space $D^{\mathcal{M}}\left(R^{1}\right)$ and by $\mathcal{D}_{\mathcal{M}}^{\prime}$ the dual space for $\mathcal{D}^{\mathcal{M}}$.

In $D^{\mathcal{M}}\left(R^{n}\right)$ we consider the following operator

$$
\varsigma_{n}^{*}: D^{\mathcal{M}}\left(R^{n}\right) \ni \varphi(t) \mapsto\left(\varsigma_{n}^{*} \circ \varphi\right)(t):=\frac{1}{n !} \sum_{\varsigma \in G_{n}} \varphi\left(t_{\varsigma(1)}, \ldots, t_{\varsigma(n)}\right),
$$

where $t=\left(t_{1}, \ldots, t_{n}\right) \in R^{n}$. The operator $\varsigma_{n}^{*}$ is a projection on the closed subspace of $D^{\mathcal{M}}\left(R^{n}\right)$ of symmetric functions

$$
\mathcal{D}^{\mathcal{M}}\left(R^{n}\right):=\mathcal{R}\left(\varsigma_{n}^{*}\right) \subset D^{\mathcal{M}}\left(R^{n}\right) .
$$

We would like to describe the dual space for $\prod_{n \in N} \mathcal{D}^{\mathcal{M}}\left(R^{n}\right)$. Let $\mathcal{D}_{\mathcal{M}}^{\prime}\left(R^{n}\right)$ denote the dual space for $\mathcal{D}^{\mathcal{M}}\left(R^{n}\right)$ with the strong topology $\beta\left\langle\mathcal{D}_{\mathcal{M}}^{\prime}\left(R^{n}\right) \mid \mathcal{D}^{\mathcal{M}}\left(R^{n}\right)\right\rangle$. We shall prove the following theorem (comp. [3]):

THEOREM 4.1. The following mappings:

$$
\begin{array}{ccccc}
\mathcal{D}_{\mathcal{M}}^{\prime}\left(R^{n}\right) & \stackrel{\varrho}{\longmapsto} \widehat{\odot}_{p}^{n} \mathcal{D}_{\mathcal{M}}^{\prime} & \stackrel{\vartheta}{\longmapsto} & \mathcal{P}_{n}\left(\mathcal{D}^{\mathcal{M}}\right) \\
T_{n} & \stackrel{\varrho}{\longmapsto} \varrho\left(T_{n}\right)=f_{n} \stackrel{\vartheta}{\longmapsto} & F_{n}
\end{array}
$$

are topological isomorphisms. Moreover the second of them

$$
\vartheta: \widehat{\odot}_{p}^{n} \mathcal{D}_{\mathcal{M}}^{\prime} \ni f_{n} \mapsto F_{n}:=f_{n} \circ \chi_{n} \circ \Delta_{n} \in \mathcal{P}_{n}\left(\mathcal{D}^{\mathcal{M}}\right)
$$

is given by the formula

$$
F_{n}(\varphi)=\left\langle f_{n} \mid \otimes^{n} \varphi\right\rangle, \quad \varphi \in \mathcal{D}^{\mathcal{M}}
$$

and is an extension $\chi_{n} \circ \Delta_{n}$ of the superposition of canonical mappings

$$
\begin{array}{ccccc}
\mathcal{D}^{\mathcal{M}} & \stackrel{\Delta_{n}}{\longrightarrow} & \prod_{i=1}^{n} \mathcal{D}^{\mathcal{M}} & \stackrel{\chi_{n}}{\longmapsto} & \otimes^{n} \mathcal{D}^{\mathcal{M}} \\
\varphi & \stackrel{\Delta_{n}}{\longmapsto} & { }^{n} \varphi & \stackrel{\chi_{n}}{\longmapsto} & \otimes^{n} \varphi
\end{array}
$$

where $\otimes^{n} \varphi$ is the scalar function of $n$ real variables,

$$
\otimes^{n} \varphi(t):=\varphi\left(t_{1}\right) \cdot \ldots \cdot \varphi\left(t_{n}\right), \quad t=\left(t_{1}, \ldots, t_{n}\right) \in R^{n} .
$$

Proof. Let operators $\varsigma_{n}$ and $\varsigma_{n}^{\prime}$ be mutually adjoint with respect to the dual pair $\left\langle\otimes^{n} \mathcal{D}_{\mathcal{M}}^{\prime} \mid \otimes^{n} \mathcal{D}^{\mathcal{M}}\right\rangle$ given by the bilinear form

$$
\left\langle u_{1} \otimes \ldots \otimes u_{n} \mid \varphi_{1} \otimes \ldots \otimes \varphi_{n}\right\rangle=\left\langle u_{1} \mid \varphi_{1}\right\rangle \ldots\left\langle u_{n} \mid \varphi_{n}\right\rangle .
$$

Then for any $u_{1}, \ldots, u_{n} \in \mathcal{D}_{\mathcal{M}}^{\prime}$ and $\varphi_{1}, \ldots, \varphi_{n} \in \mathcal{D}^{\mathcal{M}}$ the operator $\varsigma_{n}$ satisfies:

$$
\begin{aligned}
& \left\langle u_{1} \odot \ldots \odot u_{n} \mid \varphi_{1} \otimes \ldots \otimes \varphi_{n}\right\rangle=\left\langle u_{1} \otimes \ldots \otimes u_{n} \mid \varphi_{1} \odot \ldots \odot \varphi_{n}\right\rangle, \\
& \varsigma_{n}: \varphi_{1} \otimes \ldots \otimes \varphi_{n} \mapsto \varphi_{1} \odot \ldots \odot \varphi_{n}:=\frac{1}{n !} \sum_{\varsigma \in G_{n}} \varphi_{\varsigma(1)} \otimes \ldots \otimes \varphi_{\varsigma}(n) .
\end{aligned}
$$

Let $\mathcal{R}\left(\varsigma_{n}\right)$ be denoted by $\odot^{n} \mathcal{D}^{\mathcal{M}}$.

If the set of seminorms $\left\{p_{i}\right\}_{i \in I}$ defines the topology in $\mathcal{D}^{\mathcal{M}}$, then the set of seminorms

$$
\left(p_{i_{1}} \otimes \ldots \otimes p_{i_{n}}\right)(\psi)=\sum_{\psi=\sum_{m \in N_{1} n}} \inf _{\varphi_{m_{1}} \otimes \ldots \otimes \varphi_{m_{n}} \in \otimes^{n} \mathcal{D}^{\mathcal{M}}} \sum_{m \in N_{1}{ }^{n}} p_{i_{1}}\left(\varphi_{m_{1}}\right) \ldots p_{i_{n}}\left(\varphi_{m_{n}}\right)
$$


defines the projective topology in $\otimes^{n} \mathcal{D}^{\mathcal{M}}$. We have the following

$$
\begin{aligned}
\left(p_{i_{1}} \otimes \ldots \otimes p_{i_{n}}\right)\left(\varsigma_{n} \circ \psi\right) & \leq \inf \sum_{m \in N_{1} n} \frac{1}{n !} \sum_{\varsigma \in G_{n}} p_{i_{1}}\left(\varphi_{m_{\varsigma(1)}}\right) \ldots p_{i_{n}}\left(\varphi_{m_{\varsigma(n)}}\right) \\
& =\inf \sum_{m \in N_{1} n} \frac{1}{n !} \sum_{\varsigma \in G_{n}} p_{i_{\varsigma(1)}}\left(\varphi_{m_{1}}\right) \ldots p_{i_{\varsigma}(n)}\left(\varphi_{m_{n}}\right) \\
& =\varsigma_{n} \circ\left(p_{i_{1}} \otimes \ldots \otimes p_{i_{n}}\right)(\psi),
\end{aligned}
$$

where

$$
\varsigma_{n} \circ\left(p_{i_{1}} \otimes \ldots \otimes p_{i_{n}}\right):=\frac{1}{n !} \sum_{\sigma \in G_{n}} p_{i_{\varsigma(1)}} \otimes \ldots \otimes p_{i_{\varsigma}(n)}
$$

is a seminorm in $\otimes^{n} \mathcal{D}^{\mathcal{M}}$, continuous in the projective topology. Hence the projection $\varsigma_{n}$ is continuous. The continuity of $\varsigma_{n}$ and the fact that the subspace $\otimes^{n} \mathcal{D}^{\mathcal{M}}$ is dense in $\widehat{\otimes}_{p}^{n} \mathcal{D}^{\mathcal{M}}$ imply that there exists a continuous extension of $\varsigma_{n}$ on the completions of the spaces $\otimes^{n} \mathcal{D}^{\mathcal{M}}$ and $\odot{ }^{n} \mathcal{D}^{\mathcal{M}}$ respectively, namely $\varsigma_{n}: \widehat{\otimes}_{p}^{n} \mathcal{D}^{\mathcal{M}} \longrightarrow \widehat{\odot}_{p}^{n} \mathcal{D}^{\mathcal{M}}$. Hence we can represent the space $\widehat{\otimes}_{p}^{n} \mathcal{D}^{\mathcal{M}}$ as locally convex direct sum

$$
\widehat{\otimes}_{p}^{n} \mathcal{D}^{\mathcal{M}}=\widehat{\odot}_{p}^{n} \mathcal{D}^{\mathcal{M}} \dot{+} \mathcal{N}\left(\varsigma_{n}\right) .
$$

Let us remark that $\left(\widehat{\otimes}_{p}^{n} \mathcal{D}^{\mathcal{M}}\right)_{\beta}^{\prime}$ denotes the dual of $\widehat{\otimes}_{p}^{n} \mathcal{D}^{\mathcal{M}}$, endowed with the topology of uniform convergence on bounded, absolutely convex subsets, therefore one can replace the notation $\left(\widehat{\otimes}_{p}^{n} \mathcal{D}^{\mathcal{M}}\right)_{\beta}^{\prime}$ with the notation $\left(\left(\widehat{\otimes}_{p}^{n} \mathcal{D}^{\mathcal{M}}\right)^{\prime}, \tau_{\beta}\right)$.

Theorem 3.1 implies that $\mathcal{D}^{\mathcal{M}}$ is a nuclear $(D F)$-space and $\mathcal{D}_{\mathcal{M}}^{\prime}$ is a $(F)$-space. For such spaces the following isomorphism

$$
\left(\widehat{\otimes}_{p}^{n} \mathcal{D}^{\mathcal{M}}\right)_{\beta}^{\prime} \simeq \widehat{\otimes}_{p}^{n} \mathcal{D}_{\mathcal{M}}^{\prime} .
$$

holds. The isomorphism (4) implies that the bilinear form (2) defines the dual pair $\left\langle\widehat{\otimes}_{p}^{n} \mathcal{D}_{\mathcal{M}}^{\prime} \mid \widehat{\otimes}_{p}^{n} \mathcal{D}^{\mathcal{M}}\right\rangle$ and the operator $\varsigma_{n}^{\prime}$ is adjoint to $\varsigma_{n}$ with respect to this duality. In particular $\varsigma_{n}^{\prime}$ is continuous in the strong topology.

Hence, and also from the equality 3 we obtain that the space $\widehat{\otimes}_{p}^{n} \mathcal{D}_{\mathcal{M}}^{\prime}$ can be represented as the locally convex space

$$
\widehat{\otimes}_{p}^{n} \mathcal{D}_{\mathcal{M}}^{\prime}=\widehat{\odot}_{p}^{n} \mathcal{D}_{\mathcal{M}}^{\prime}+\mathcal{N}\left(\varsigma_{n}^{\prime}\right) .
$$

When in the space $\mathcal{L}^{n}\left(\mathcal{D}^{\mathcal{M}}, C\right)$ the topology $\tau_{\beta}$ of uniform convergence on bounded absolutely convex subsets is considered we have that

$$
\left(\mathcal{L}^{n}\left(\mathcal{D}^{\mathcal{M}}, C\right), \tau_{\beta}\right) \simeq\left(\widehat{\otimes}_{p}^{n} \mathcal{D}^{\mathcal{M}}\right)_{\beta}^{\prime}
$$

Then from (4), (5) and (6) we get that

$$
\left(\mathcal{L}^{n}\left(\mathcal{D}^{\mathcal{M}}, C\right), \tau_{\beta}\right) \simeq \widehat{\otimes}_{p}^{n} \mathcal{D}_{\mathcal{M}}^{\prime} \simeq \widehat{\odot}_{p}^{n} \mathcal{D}_{\mathcal{M}}^{\prime}+\mathcal{N}\left(\varsigma_{n}^{\prime}\right)
$$

The first isomorphism in (7) implies, in particular, that any form $\bar{f}_{n} \in \mathcal{L}^{n}\left(\mathcal{D}^{\mathcal{M}}, C\right)$ can be represented as $\bar{f}_{n}=\bar{F}_{n} \circ \chi_{n}$ for some $\bar{F}_{n} \in \widehat{\otimes}_{p}^{n} \mathcal{D}_{\mathcal{M}}^{\prime}$, and there exists the representation in the form of absolutely convergent series $\bar{F}_{n}=\sum_{l \in N_{1}{ }^{n}} u_{l_{1}} \otimes \ldots \otimes u_{l_{n}} \in \widehat{\otimes}_{p}^{n} \mathcal{D}_{\mathcal{M}}^{\prime}$, where $u_{l_{\iota}} \in \mathcal{D}_{\mathcal{M}}^{\prime}, \iota=1, \ldots, n\left[10\right.$, Theorem 6.4]. Hence for any $\varphi_{1}, \ldots, \varphi_{n} \in \mathcal{D}^{\mathcal{M}}$ the operator 
$\varsigma_{n}^{\prime}$ satisfies the following equalities

$$
\begin{aligned}
\left(\varsigma_{n}^{\prime} \circ \bar{f}_{n}\right)\left(\varphi_{1}, \ldots, \varphi_{n}\right) & =\frac{1}{n !} \sum_{\varsigma \in G_{n}} \sum_{l \in N_{1} n}\left\langle u_{l_{\varsigma(1)}} \mid \varphi_{1}\right\rangle \ldots\left\langle u_{l_{\varsigma(n)}} \mid \varphi_{n}\right\rangle \\
& =\frac{1}{n !} \sum_{\varsigma \in G_{n}} \sum_{l \in N_{1} n}\left\langle u_{l_{1}} \mid \varphi_{\varsigma(1)}\right\rangle \ldots\left\langle u_{l_{n}} \mid \varphi_{\varsigma(n)}\right\rangle,
\end{aligned}
$$

which means that the composition $f_{n}^{\varsigma}:=\varsigma_{n}^{\prime} \circ \bar{f}_{n}$ belongs to the space $\mathcal{L}_{\varsigma}^{n}\left(\mathcal{D}^{\mathcal{M}}, C\right)$ of symmetric continuous $n$-linear forms on $\mathcal{D}^{\mathcal{M}}$. The second isomorphism in (7) implies

$$
\mathcal{R}\left(\varsigma_{n}^{\prime}\right)=\left(\mathcal{L}_{\varsigma}^{n}\left(\mathcal{D}^{\mathcal{M}}, C\right), \tau_{\beta}\right) \simeq \widehat{\odot}_{p}^{n} \mathcal{D}_{\mathcal{M}}^{\prime}
$$

Now we shall prove that the following topological isomorphism

$$
\left(\mathcal{L}_{\varsigma}^{n}\left(\mathcal{D}^{\mathcal{M}}, C\right), \tau_{\beta}\right) \simeq \mathcal{P}_{n}\left(\mathcal{D}^{\mathcal{M}}\right)
$$

holds. For any symmetric form $f_{n}^{\varsigma} \in \mathcal{L}_{\varsigma}^{n}\left(\mathcal{D}^{\mathcal{M}}, C\right)$ we have the polarization formula (1). Hence its restriction to the diagonal of cartesian product

$$
\Delta_{n}^{\prime}:\left(\mathcal{L}_{\varsigma}^{n}\left(\mathcal{D}^{\mathcal{M}}, C\right), \tau_{\beta}\right) \ni f_{n}^{\varsigma} \longrightarrow f_{n}^{\varsigma} \circ \Delta_{n} \in \mathcal{P}_{n}\left(\mathcal{D}^{\mathcal{M}}\right)
$$

should be the isomorphism $(9)$ we are looking for. Since $\Delta_{n}^{\prime}$ is surjective, then it is enough to prove its continuity. Any continuous seminorm on $\mathcal{P}_{n}\left(\mathcal{D}^{\mathcal{M}}\right)$ has the form

$$
p_{S}\left(f_{n}^{\varsigma} \circ \Delta_{n}\right)=\sup _{\varphi \in S}\left|\left(f_{n}^{\varsigma} \circ \Delta_{n}\right)(\varphi)\right|, \quad f_{n}^{\varsigma} \circ \Delta_{n} \in \mathcal{P}_{n}\left(\mathcal{D}^{\mathcal{M}}\right),
$$

where $S$ is a bounded absolutely convex subset of $\mathcal{D}^{\mathcal{M}}$. The polarization formula (1) implies that

$$
\begin{aligned}
p_{S_{1} \ldots S_{n}}\left(f_{n}^{\varsigma}\right) & \leq \frac{1}{2^{n} \cdot n !} \sum_{e_{\iota}= \pm 1} \sup _{\iota \in\{1, \ldots, n\}} \sup _{\varphi_{\iota} \in S_{\iota}}\left|\left(f_{n}^{\varsigma} \circ \Delta_{n}\right)\left(\sum_{\iota=1}^{n} e_{\iota} \varphi_{\iota}\right)\right| \\
& =\frac{n^{n}}{2^{n} \cdot n !} \sum_{e_{\iota}= \pm 1} \sup _{\iota \in\{1, \ldots, n\}} \sup _{x_{\iota} \in S_{\iota}}\left|\left(f_{n}^{\varsigma} \circ \Delta_{n}\right)\left(\frac{1}{n} \sum_{\iota=1}^{n} e_{\iota} \varphi_{\iota}\right)\right| \\
& \leq \frac{n^{n}}{n !} p_{S}\left(f_{n}^{\varsigma} \circ \Delta_{n}\right) .
\end{aligned}
$$

Hence $\Delta_{n}^{\prime}$ is the required isomorphism (9).

By combining the isomorphisms (8) and (9) we obtain that the mapping

$$
\widehat{\odot}_{p}^{n} \mathcal{D}_{\mathcal{M}}^{\prime} \ni f_{n} \mapsto\left\langle f_{n} \mid \otimes^{n} \varphi\right\rangle=\left(f_{n} \circ \chi_{n} \circ \Delta_{n}\right)(\varphi):=F_{n}(\varphi),
$$

given for any $\varphi \in \mathcal{D}^{\mathcal{M}}$, is the second isomorphism $\vartheta$. It is known [5, Theorem 2.1] that

$$
D^{\mathcal{M}}\left(R^{n}\right) \simeq \widehat{\otimes}_{p}^{n} \mathcal{D}^{\mathcal{M}} \text {. }
$$

Isomorphism (11) implies that the functions of the form $\varphi(t)=\sum_{l \in N_{1}^{n}} \varphi_{l_{1}}\left(t_{1}\right) \ldots \varphi_{l_{n}}\left(t_{n}\right)$, where $\varphi_{l_{1}}, \ldots, \varphi_{l_{n}} \in \mathcal{D}^{\mathcal{M}}$, form a dense subspace $\otimes^{n} \mathcal{D}^{\mathcal{M}}$ of the space $D^{\mathcal{M}}\left(R^{n}\right)$. Since

$$
\begin{aligned}
\left(\varsigma_{n}^{*} \circ \varphi\right)(t) & =\frac{1}{n !} \sum_{l \in N_{1}{ }^{n}} \sum_{\varsigma \in G_{n}} \varphi_{l_{1}}\left(t_{\varsigma(1)}\right) \ldots \varphi_{l_{n}}\left(t_{\varsigma(n)}\right) \\
& =\frac{1}{n !} \sum_{l \in N_{1}{ }^{n}} \sum_{\varsigma \in G_{n}} \varphi_{l_{\varsigma(1)}}\left(t_{1}\right) \ldots \varphi_{l_{\varsigma(n)}}\left(t_{n}\right)=\left(\varsigma_{n} \circ \varphi\right)(t),
\end{aligned}
$$


the continuity of projections implies that the algebraic equality

$$
\mathcal{D}^{\mathcal{M}}\left(R^{n}\right)=\widehat{\odot}_{p}^{n} \mathcal{D}^{\mathcal{M}}
$$

holds. The topological isomorphism $\mathcal{D}^{\mathcal{M}}\left(R^{n}\right) \simeq \widehat{\odot}_{p}^{n} \mathcal{D}^{\mathcal{M}}$ is a corollary of 11 and the adjoint topological isomorphism

$$
\varrho: \mathcal{D}_{\mathcal{M}}^{\prime}\left(R^{n}\right) \longrightarrow \widehat{\odot}_{p}^{n} \mathcal{D}_{\mathcal{M}}^{\prime}
$$

is obvious.

Let us denote

$$
\mathcal{D}_{S}^{\prime}:=\sum_{n \in N_{1}} \mathcal{D}_{\mathcal{M}}^{\prime}\left(R^{n}\right), \quad \mathcal{D}_{S}^{\mathcal{M}}:=\prod_{n \in N_{1}} \mathcal{D}^{\mathcal{M}}\left(R^{n}\right)
$$

Notice that $\left\langle\mathcal{D}_{S}^{\prime} \mid \mathcal{D}_{S}^{\mathcal{M}}\right\rangle$ is a dual pair according to its canonical bilinear form

$$
\langle T \mid \bar{\varphi}\rangle=\sum_{n \in N_{1}}\left\langle T_{n} \mid \varphi_{n}\right\rangle \quad \text { for } \quad T=\sum_{n \in N_{1}} T_{n} \in \mathcal{D}_{S}^{\prime}, \quad \bar{\varphi}=\prod_{n \in N_{1}} \varphi_{n} \in \mathcal{D}_{S}^{\mathcal{M}},
$$

where $T_{n} \in \mathcal{D}_{\mathcal{M}}^{\prime}\left(R^{n}\right)$ and $\varphi_{n} \in \mathcal{D}^{\mathcal{M}}\left(R^{n}\right)$. Let us remark that if $\bar{\varphi} \in \mathcal{D}_{S}^{\mathcal{M}}$, then $\bar{\varphi}=\left(\varphi_{n}\right)$ and, for different $k$ and $n, \varphi_{k}$ is a function of $\left(x^{k}{ }_{1}, x^{k}{ }_{2}, \ldots, x_{k}^{k}\right)$ and $\varphi_{n}$ is a function of $\left(x^{n}{ }_{1}, x^{n}{ }_{2}, \ldots, x_{n}^{n}\right)$, where $\left(x^{k}{ }_{1}, x^{k}{ }_{2}, \ldots, x^{k} \min (k, n)\right)$ and $\left(x^{n}{ }_{1}, x^{n}{ }_{2}, \ldots, x^{n}{ }_{\min (k, n)}\right)$ can be different.

We shall prove the following

\section{THEOREM 4.2 .}

(i) The locally convex space $\sum_{n \in N_{1}} \widehat{\odot}_{p}^{n} \mathcal{D}_{\mathcal{M}}^{\prime}$ is a topological algebra with respect to convolution, given by the formula

$$
f * h:=\sum_{n \in N_{1}}\left(\sum_{m=1}^{n} f_{m} \odot h_{n-m+1}\right)
$$

where $f=\sum_{n \in N_{1}} f_{n}, h=\sum_{n \in N_{1}} h_{n} \in \sum_{n \in N_{1}} \widehat{\odot}_{p}^{n} \mathcal{D}_{\mathcal{M}}^{\prime}$ and $f_{n}, h_{n} \in \widehat{\odot}_{p}^{n} \mathcal{D}_{\mathcal{M}}^{\prime}$.

(ii) The following mappings:

$$
\begin{array}{cl}
\mathcal{D}_{S}^{\prime} & \stackrel{\varrho}{\longmapsto} \sum_{n \in N_{1}} \widehat{\odot}_{p}^{n} \mathcal{D}_{\mathcal{M}}^{\prime} \stackrel{\vartheta}{\longmapsto} \mathcal{P}\left(\mathcal{D}^{\mathcal{M}}\right) \\
T=\sum_{n \in N_{1}} T_{n} \stackrel{\varrho}{\longmapsto} f=\sum_{n \in N_{1}} f_{n} \stackrel{\vartheta}{\longmapsto} F=\sum_{n \in N_{1}} F_{n}
\end{array}
$$

where $f_{n}:=\varrho\left(T_{n}\right)$ and $F_{n}:=f_{n} \circ \chi_{n} \circ \Delta_{n}=\vartheta\left(f_{n}\right)$, are surjective topological isomorphisms.

(iii) The convolution in the algebra $\sum_{n \in N_{1}} \widehat{\odot}_{p}^{n} \mathcal{D}_{\mathcal{M}}^{\prime}$ is transformed by the isomorphism $\vartheta$ into the product of polynomials in the algebra $\mathcal{P}\left(\mathcal{D}^{\mathcal{M}}\right)$, i.e.

$$
\vartheta(f * h)=F \cdot H, \quad F=\vartheta(f), \quad H=\vartheta(h) \in \mathcal{P}\left(\mathcal{D}^{\mathcal{M}}\right) .
$$

Proof. If we put

$$
\varrho(T)=\sum_{n \in N_{1}} \varrho\left(T_{n}\right)=\sum_{n \in N_{1}} f_{n}=f, \quad \vartheta(f)=\sum_{n \in N_{1}} \vartheta\left(f_{n}\right)=\sum_{n \in N_{1}} F_{n}=F,
$$

then Theorem 4.1 implies that there exist isomorphisms $\varrho$ and $\vartheta$. 
Now we prove (i); for any $f_{n} \in \widehat{\odot}_{p}^{n} \mathcal{D}_{\mathcal{M}}^{\prime}$ and $h_{m} \in \widehat{\odot}_{p}^{m} \mathcal{D}_{\mathcal{M}}^{\prime}$ we have that

$$
f_{n} \odot h_{m} \in\left(\widehat{\odot}_{p}^{n} \mathcal{D}_{\mathcal{M}}^{\prime}\right) \odot\left(\widehat{\odot}_{p}^{m} \mathcal{D}_{\mathcal{M}}^{\prime}\right) \subset \widehat{\odot}_{p}^{n+m} \mathcal{D}_{\mathcal{M}}^{\prime}
$$

and the convolution " $*$ " in the direct sum $\sum_{n \in N_{1}} \widehat{\odot}_{p}^{n} \mathcal{D}_{\mathcal{M}}^{\prime}$ is well defined. Its continuity follows from the continuity of the canonical mapping in the symmetric tensor product

$$
\left(\widehat{\odot}_{p}^{n} \mathcal{D}_{\mathcal{M}}^{\prime}\right) \times\left(\widehat{\odot}_{p}^{m} \mathcal{D}_{\mathcal{M}}^{\prime}\right) \ni\left(f_{n}, h_{m}\right) \rightarrow f_{n} \odot h_{m} \in \widehat{\odot}_{p}^{n+m} \mathcal{D}_{\mathcal{M}}^{\prime} .
$$

From the formula 10 we obtain that

$$
\begin{aligned}
F_{n}(\varphi) \cdot H_{m}(\varphi) & =\left\langle f_{n} \mid \otimes^{n} \varphi\right\rangle \cdot\left\langle h_{m} \mid \otimes^{m} \varphi\right\rangle=\left\langle f_{n} \otimes h_{m} \mid \otimes^{n+m} \varphi\right\rangle \\
& =\left\langle f_{n} \odot h_{m} \mid \otimes^{n+m} \varphi\right\rangle=\left(f_{n} \odot h_{m}\right) \circ \chi_{n+m} \circ \Delta_{n+m}(\varphi) .
\end{aligned}
$$

Hence $F_{n} \cdot H_{m} \in \mathcal{P}_{n+m}\left(\mathcal{D}^{\mathcal{M}}\right)$ and for any polynomial ultradistributions $F=\sum_{n \in N_{1}} F_{n}$ and $H=\sum_{n \in N_{1}} H_{n}$ belonging to the space $\mathcal{P}\left(\mathcal{D}^{\mathcal{M}}\right)$ we get that

$$
\begin{aligned}
F(\varphi) \cdot H(\varphi) & =\sum_{n \in N_{1}} \sum_{m=1}^{n} F_{m}(\varphi) \cdot H_{n-m+1}(\varphi) \\
& =\sum_{n \in N_{1}} \sum_{m=1}^{n}\left(f_{m} \odot h_{n-m+1}\right) \circ \chi_{n+1} \circ \Delta_{n+1}(\varphi) \\
& =(f * h) \circ \chi_{n+1} \circ \Delta_{n+1}(\varphi) .
\end{aligned}
$$

Therefore the mapping

$$
\sum_{n \in N_{1}} \widehat{\odot}_{p}^{n} \mathcal{D}_{\mathcal{M}}^{\prime} \ni f=\sum_{n \in N_{1}} f_{n} \stackrel{\vartheta}{\longmapsto} F=\sum_{n \in N_{1}} f_{n} \circ \chi_{n} \circ \Delta_{n} \in \mathcal{P}\left(\mathcal{D}^{\mathcal{M}}\right)
$$

transforms the convolution into the product of polynomial ultradistributions.

5. Entire functions of exponential type. Let now $\nu=\left(\nu_{1}, \ldots, \nu_{n}\right)$ be an arbitrarily chosen vector with positive coordinates and let $a=\left(a_{1}, \ldots, a_{n}\right), b=\left(b_{1}, \ldots, b_{n}\right) \in R^{n}$ be such that $b \succ a$. Let $\zeta=\left(\zeta_{1}, \ldots, \zeta_{n}\right) \in C^{n}$ and $\zeta=\xi+\mathrm{i} \tau$, where $\xi=\left(\xi_{1}, \ldots, \xi_{n}\right)$, $\tau=\left(\tau_{1}, \ldots, \tau_{n}\right) \in R^{n}$. In the space of entire functions we introduce the subspace of functions of exponential type in the following way

$$
E_{\nu}[a, b]=\left\{\Phi: C^{n} \ni \zeta \mapsto \Phi(\zeta) \in C, \quad\|\Phi\|_{E_{\nu}[a, b]}<\infty\right\},
$$

with the norm given by the formula

$$
\|\Phi\|_{E_{\nu}[a, b]}=\sup _{k \in N^{n}} \sup _{\zeta \in C^{n}} \frac{\left|\zeta^{k} \Phi(\zeta) \exp \left(-H_{[a, b]}(\tau)\right)\right|}{\nu^{k} \mu_{k}}
$$

where for $t=\left(t_{1}, \ldots, t_{n}\right), \tau=\left(\tau_{1}, \ldots, \tau_{n}\right) \in R^{n}$

$$
H_{[a, b]}(\tau)=\sup _{t \in[a, b]}(t, \tau), \quad(t, \tau)=\sum_{\iota=1}^{n} t_{\iota} \tau_{\iota}
$$

is the supporting function of the $n$-dimensional cube $[a, b] \subset R^{n}$. We call

$$
E\left(C^{n}\right):=\bigcup\left\{E_{\nu}[a, b]: \nu \in \operatorname{int} R_{+}^{n}, \quad[a, b] \subset R^{n}\right\}
$$

the space of ultraincreasing functions of exponential type. 
Let us notice that $E\left(C^{n}\right)$ is contained in the known locally convex space of entire functions of exponential type described for example in [6, 9.1] and [8].

The following theorem gives some connection between $D^{\mathcal{M}}\left(R^{n}\right)$ and $E\left(C^{n}\right)$.

TheOREM 5.1 ([4, Theorem 9.1]). The Fourier transform is a surjective topological isomorphism

$$
\mathcal{F}: D^{\mathcal{M}}\left(R^{n}\right) \rightarrow E\left(C^{n}\right) .
$$

The adjoint Fourier transform is a topological isomorphism of dual spaces, endowed with their strong topologies

$$
\mathcal{F}^{\prime}: E^{\prime}\left(C^{n}\right) \rightarrow D_{\mathcal{M}}^{\prime}\left(R^{n}\right)
$$

Theorems 3.1 and 5.1 imply in particular that the spaces $E\left(C^{n}\right)$ and $E^{\prime}\left(C^{n}\right)$ are nontrivial, nuclear, reflexive, locally convex. Moreover $E\left(C^{n}\right)$ is a $\left(L N^{*}\right)$-space in the sense of Silva and (DF)-space and $E^{\prime}\left(C^{n}\right)$ is a $\left(M^{*}\right)$-space in the sense of Silva.

Let $E=E(C)$ denote the space of ultraincreasing entire functions of exponential type of one complex variable. We shall prove a Paley-Wiener type theorem for polynomial ultradistributions.

THEOREM 5.2. The Fourier transform

$$
\mathcal{F}: \mathcal{D}^{\mathcal{M}} \rightarrow E
$$

can be unambiguously extended to the topological isomorphism

$$
\overline{\mathcal{F}}_{\mathcal{P}}^{\prime}: \mathcal{P}(E) \rightarrow \mathcal{P}\left(\mathcal{D}^{\mathcal{M}}\right)
$$

The proof of this theorem is postponed after Theorem 5.3 .

The range of the projection

$$
\left(\sigma_{n} \circ \Phi\right)(z)=\frac{1}{n !} \sum_{\varsigma \in G_{n}} \Phi\left(z_{\varsigma(1)}, \ldots, z_{\varsigma(n)}\right), \quad \Phi \in E\left(C^{n}\right)
$$

is denoted by $\mathcal{E}\left(C^{n}\right)=\mathcal{R}\left(\sigma_{n}\right)$. Obviously $\sigma_{1}(E)=E$. Let $\mathcal{E}^{\prime}\left(C^{n}\right)$ be the strong dual space to $\mathcal{E}\left(C^{n}\right)$.

From Theorems 4.1 and 5.1 the linear topological isomorphisms

$$
\mathcal{E}^{\prime}\left(C^{n}\right) \simeq \widehat{\odot}_{p}^{n} E^{\prime} \simeq \mathcal{P}_{n}(E)
$$

follow. The symmetric projective tensor product $\odot_{p}^{n} E^{\prime}$ and the space of polynomials $\mathcal{P}_{n}(E)$ are understood in the same way as $\odot_{p}^{n} \mathcal{D}_{\mathcal{M}}^{\prime}$ and $\mathcal{P}_{n}\left(\mathcal{D}^{\mathcal{M}}\right)$. The second of these isomorphisms is determined by the formula

$$
\odot_{p}^{n} E^{\prime} \ni \widetilde{P}_{n} \mapsto\left\langle\left.\widetilde{P}_{n}\right|^{n} \Phi\right\rangle=P_{n}(\Phi) \in \mathcal{P}_{n}(E), \quad \Phi \in E .
$$

Let us denote

$$
\mathcal{E}^{\prime}=\sum_{n \in N_{1}} \mathcal{E}^{\prime}\left(C^{n}\right), \quad \mathcal{E}=\prod_{n \in N_{1}} \mathcal{E}\left(C^{n}\right) .
$$

The following theorem is true: 
THEOREM 5.3. The following mappings:

$$
\begin{array}{cl}
\mathcal{E}^{\prime} & \stackrel{\widetilde{\varrho}}{\longrightarrow} \sum_{n \in N_{1}} \widehat{\odot}_{p}^{n} E^{\prime} \stackrel{\widetilde{\vartheta}}{\longrightarrow} \mathcal{P}(E) \\
T=\sum_{n \in N_{1}} T_{n} \stackrel{\widetilde{\varrho}}{\longmapsto} f=\sum_{n \in N_{1}} f_{n} \stackrel{\widetilde{\vartheta}}{\longmapsto} F=\sum_{n \in N_{1}} F_{n},
\end{array}
$$

where $f_{n}=\widetilde{\varrho}\left(T_{n}\right)$ and $F_{n}=f_{n} \circ \widetilde{\chi}_{n} \circ \widetilde{\Delta}_{n}=\widetilde{\vartheta}\left(f_{n}\right)$, are topological isomorphisms.

Proof. The isomorphisms $\widetilde{\varrho}, \widetilde{\vartheta}$ exist from 12 if we put

$$
\widetilde{\vartheta}(f)=\sum_{n \in N_{1}} \widetilde{\vartheta}\left(f_{n}\right)=\sum_{n \in N_{1}} F_{n}=F
$$

which completes the proof.

Proof of Theorem 5.2. Let $\mathcal{F}^{\prime}: E^{\prime} \longrightarrow \mathcal{D}_{\mathcal{M}}^{\prime}$ denote the adjoint Fourier transform with respect to dual pairs $\left\langle E^{\prime} \mid E\right\rangle$ and $\left\langle\mathcal{D}_{\mathcal{M}}^{\prime} \mid \mathcal{D}^{\mathcal{M}}\right\rangle$ and let the operator $\overline{\mathcal{F}}^{\prime}$ be defined in the following way

$$
\begin{aligned}
\overline{\mathcal{F}}_{\Pi}^{\prime}:=\prod_{n=1}^{\infty}{ }^{n} \mathcal{F}^{\prime}: & \sum_{n \in N_{1}} \widehat{\otimes}_{p}^{n} E^{\prime} \\
f=\sum_{n \in N_{1}} f_{n} & \mapsto \sum_{n \in N_{1}} \widehat{\otimes}_{p}^{n} \mathcal{D}_{\mathcal{M}}^{\prime} f=\sum_{n \in N_{1}}{ }^{\prime} \mathcal{F}^{\prime} f_{n},
\end{aligned}
$$

where

$$
\begin{aligned}
{ }^{n} \mathcal{F}^{\prime}:=\underbrace{\mathcal{F}^{\prime} \otimes \ldots \otimes \mathcal{F}^{\prime}}_{n}: & \widehat{\otimes}_{p}^{n} E^{\prime} \\
v_{1} \otimes \ldots \otimes v_{n} & \mapsto \widehat{\otimes}_{p}^{n} \mathcal{D}_{\mathcal{M}}^{\prime}, \\
& \mathcal{F}^{\prime} v_{1} \otimes \ldots \otimes \mathcal{F}^{\prime} v_{n} .
\end{aligned}
$$

Theorem 5.1 implies that

$$
\mathcal{N}\left(\mathcal{F}^{\prime}\right)=\{0\}, \quad \mathcal{R}\left(\mathcal{F}^{\prime}\right)=\mathcal{D}_{\mathcal{M}}^{\prime}
$$

For nuclear spaces $X$ and $Y$ and for a linear, continuous operator $A: X \rightarrow X$ it is true that $\mathcal{N}\left(A \otimes I_{Y}\right)=\mathcal{N}(A) \widehat{\otimes}_{p} Y$ (comp. Lemma 9 of [7]). One can also prove that $\mathcal{N}\left(I_{X} \otimes B\right)=X \widehat{\otimes}_{p} \mathcal{N}(B)$, when $B$ is a linear continuous operator in $Y$. Since the spaces considered are nuclear and $(13)$ holds, then

$$
\mathcal{N}\left({ }^{n} \mathcal{F}^{\prime}\right)=\{0\}, \quad \overline{\mathcal{R}\left(n \mathcal{F}^{\prime}\right)} \simeq \widehat{\otimes}_{p}^{n} \mathcal{D}_{\mathcal{M}}^{\prime},
$$

hence the mapping ${ }^{n} \mathcal{F}^{\prime}$ is a continuous isomorphism with dense image. The inverse mapping is of the form

$$
\left({ }^{n} \mathcal{F}^{\prime}\right)^{-1}:=\underbrace{\left(\mathcal{F}^{\prime}\right)^{-1} \otimes \ldots \otimes\left(\mathcal{F}^{\prime}\right)^{-1}}_{n}: \widehat{\otimes}_{p}^{n} \mathcal{D}_{\mathcal{M}}^{\prime} \longrightarrow \widehat{\otimes}_{p}^{n} E^{\prime}
$$

and it is continuous as a tensor product of continuous operators. Therefore its domain is equal to $\overline{\mathcal{R}\left({ }^{n} \mathcal{F}^{\prime}\right)}=\mathcal{R}\left({ }^{n} \mathcal{F}^{\prime}\right)=\widehat{\otimes}_{p}^{n} \mathcal{D}_{\mathcal{M}}^{\prime}$. Hence we obtain that $\overline{\mathcal{F}}^{\prime}$ is also a topological isomorphism.

From the definitions of the relevant mappings we have isomorphisms

$$
\widehat{\odot}_{p}^{n} E^{\prime} \stackrel{n}{\mathcal{F}^{\prime}} \widehat{\odot}_{p}^{n} \mathcal{D}_{\mathcal{M}}^{\prime}, \quad \sum_{n \in N_{1}} \widehat{\odot}_{p}^{n} E^{\prime} \stackrel{\overline{\mathcal{F}}^{\prime}}{\simeq} \sum_{n \in N_{1}} \widehat{\odot}_{p}^{n} \mathcal{D}_{\mathcal{M}}^{\prime} .
$$


Indeed, the composition $\varsigma_{n}^{\prime} \circ^{n} \mathcal{F}^{\prime}$ (where $\varsigma_{n}$ denotes the symmetrization operator) transforms $\widehat{\odot}_{p}^{n} E^{\prime}$ into $\widehat{\odot}_{p}^{n} \mathcal{D}_{\mathcal{M}}^{\prime}$ and because $\odot^{n} \mathcal{D}_{\mathcal{M}}^{\prime} \subset \mathcal{R}\left(\varsigma_{n}^{\prime} \circ{ }^{n} \mathcal{F}^{\prime}\right)$, the image of this composition is dense in $\widehat{\odot}_{p}^{n} \mathcal{D}_{\mathcal{M}}^{\prime}$. The inverse mapping has the form $\left({ }^{n} \mathcal{F}^{\prime}\right)^{-1} \circ \varsigma_{n}^{\prime}$ and it is defined on $\mathcal{R}\left(\varsigma_{n}^{\prime} \circ{ }^{n} \mathcal{F}^{\prime}\right)$. The continuity of this mapping implies that there exists an extension of it on $\widehat{\odot}_{p}^{n} \mathcal{D}_{\mathcal{M}}^{\prime}$, hence $\mathcal{R}\left(\varsigma_{n}^{\prime} \circ{ }^{n} \mathcal{F}^{\prime}\right)=\widehat{\odot}_{p}^{n} \mathcal{D}_{\mathcal{M}}^{\prime}$.

Since the diagram

$$
\begin{gathered}
\mathcal{P}\left(\mathcal{D}^{\mathcal{M}}\right) \stackrel{\vartheta^{-1}}{\longrightarrow} \sum \widehat{\odot}_{p}^{n} \mathcal{D}_{\mathcal{M}}^{\prime} \\
\overline{\mathcal{F}}_{\mathcal{P}}^{\prime} \downarrow \quad \downarrow \overline{\mathcal{F}}^{\prime-1} \\
\mathcal{P}(E) \stackrel{\widetilde{\vartheta}^{-1}}{\longrightarrow} \sum \widehat{\odot}_{p}^{n} E^{\prime}
\end{gathered}
$$

should commute, the operator $\mathcal{F}_{\mathcal{P}}^{\prime}$ is unambiguously defined and it is a topological isomorphism.

\section{References}

[1] H.-J. Borchers, Algebras of unbounded operators in quantum fields theory, Phys. A 124 (1984), 127-144.

[2] S. Dineen, Complex Analysis on Infinite Dimensional Spaces, Springer, 1999.

[3] K. Grasela, Generalized derivations and Fourier transform of polynomial ulradistributions, Matematychni Studii 20 (2002), 167-178.

[4] H. Komatsu, Ultradistributions I. Structure theorems and a characterization, J. Fac. Sci. Tokyo, Sect. IA 20 (1973), 25-105.

[5] H. Komatsu, Ultradistributions II. The kernel theorem and ultradistributions with support in a submanifold, J. Fac. Sci. Univ. Tokyo, Sect. IA Math. 24 (1977), 607-628.

[6] P. Lelong and L. Gruman, Entire Functions of Several Complex Variables, Springer, Berlin, 1986.

[7] B. S. Mityagin, Nucléarité et autres propriétés des espaces de type s, Trudy Mosk. Mat. Ob-va 9 (1960), 317-328.

[8] V. V. Napalkov, Convolution Equations in Several Variables Spaces, Nauka, Moscow, 1982.

[9] C. Roumieu, Sur quelques extensions de la notion de distribution, Ann. Sci. École Norm. Sup. 77 (1960), 41-121.

[10] H. Schaefer, Topological Vector Spaces, Springer, 1971. 
